\title{
Determination of the true water content, the nature of the impurities and their concentration in transformer oil
}

\author{
I $D$ Gizzatova*, $V K$ Kozlov and $D M$ Valiullina
}

Kazan State Power Engineering University, Russian Federation

\begin{abstract}
During the operation of transformers, the insulating oil is aging, therefore, there is the deterioration of its insulating properties, which is determined by multiple physico-chemical processes. The most dangerous factor, from the point of view of preserving the operational reliability and resource of a power transformer, is the moisture content of the insulation. Most methods for determining moisture content have measurement errors. These errors are largely dependent on impurities that are contained in the oil. The modified Fisher method proposed in the article allows determining the amount of impurities in the oil and the absolute moisture content. Conducted research allows also to determine the composition of impurities, to reveal the relationship between impurities and some parameters of transformer oil.
\end{abstract}

\section{Introduction}

Insulating oils are used in electric power industry as an insulating and cooling habitat in power transformers, instrument transformers, switches and other devices.

In oil-filled equipment, the oil works under difficult conditions: high electric field strength, small insulating gaps, high temperature, etc. In electric devices, oil comes in contact with chemically active metals (copper, iron, etc.). This accelerates the aging of the liquid insulation, causes a change in its chemical composition, as a result of which the electrical insulating properties of the oil deteriorate and damage to the device may occur. In order to prevent such a situation in energy systems, periodic monitoring of the insulating oil state is carried out [1-4].

One of the important indicators of the quality of insulating oil is its moisture content. The most common method for determining moisture content in transformer oil is the standardized Karl Fisher titration method [5-8]. In this method, despite its widespread use, there is a significant shortcoming - its ambiguity for oxidized oils, since Fisher's reagent interacts with oxidation products containing the $\mathrm{OH}$ group. Interfering substances can be divided into the following groups: oxidizers, reductants, basic oxides, hydroxides and salts.

To eliminate the influence of interfering components (impurities) on the determination of moisture content, we have proposed several options for improving the accuracy of the Fisher method.

It is not possible to remove interfering components from used transformer oil. But you can determine the moisture content in transformer oil before drying and after it. The difference between these measurements is the water content in the oil without the influence of impurities.
A vacuum drying unit for transformer oil was created, which consists of an $831 \mathrm{KF}$ coulometric titrator and a vacuum pump. Transformer oil $(100 \mathrm{~g})$ in the flask is heated to a temperature of $100^{\circ} \mathrm{C}$. Using a vacuum pump $(\mathrm{P}=2 \mathrm{~mm} \mathrm{Hg})$ connected to the flask, a vacuum is created and moisture is removed. The flask is uniformly continuously heated throughout the experiment, at equal intervals the experimental dried transformer oil is taken from the flask and its moisture content is determined using a coulometric titrator $831 \mathrm{KF}$ Coulometer (the weight of the introduced sample of oil in the device is $10 \mathrm{~g})[9]$.

The method of removing water from the oil can be keeping the oil at low temperatures. Having determined the moisture before cooling using a coulometric titrator, we put a sample of the oil in the freezer at a temperature of $-18^{\circ} \mathrm{C}$ and hold it for 12 hours. In the process of cooling the water crystallizes, we get dry oil, therefore, we observe the complete removal of moisture in the taken sample.

The moisture can be removed in the process of heating the oil. Samples of transformer oil were subjected to microwave radiation (SHF radiation) in a microwave oven $(\mathrm{P}=1000 \mathrm{~W})$ for 40 minutes. During the experiment, the temperature of the oil increased, the water in it evaporated.

Thus, before evacuating, cooling or heating transformer oil, the device shows the total value of moisture and impurities contained in the oil, and after applying one of the methods for removing water it determines only the amount of impurities, because water is removed and the reagent interacts with the oxidized forms remaining in the oil.

The data of experimental studies are presented in table 1.

\footnotetext{
* Corresponding author: irina-zag@bk.ru
} 
Table 1. The results of an experimental study of transformer oil samples.

\begin{tabular}{|c|c|c|c}
\hline № of sample & $\begin{array}{c}\text { Moisture content before the } \\
\text { experiment, } \mathrm{ppm}\end{array}$ & $\begin{array}{c}\text { Absolute moisture } \\
\text { content, } \mathrm{ppm}\end{array}$ & $\begin{array}{c}\text { Moisture content after the experiment (amoun } \\
\text { of impurities), ppm }\end{array}$ \\
\hline 1 & 29.7 & 10.4 & 19.3 \\
\hline 2 & 30.2 & 11.1 & 19.1 \\
\hline 3 & 25.7 & 11.9 & 13.8 \\
\hline 4 & 27.3 & 12.1 & 15.2 \\
\hline
\end{tabular}

Conducted research also allows to determine the composition of impurities. Since they affect the electrophysical parameters of the oil, it is possible to identify the dependence between impurities and some parameters of the oil [10].

Consider the dependence of the amount of impurities on one of the chemical properties of transformer oil - the acid number. The higher the acid number, the more impurities are contained in the oil. Oxides and hydroxides of some metals form iodides and react to Fisher's reagent in the same way as water. The transformer oil acid number should be within the normal range (not more than $0.25 \mathrm{mgKOH} / \mathrm{g}$ ) [11]. Figure 1 shows the dependence of the impurity concentration on the acid number, which has a linear dependence. Therefore, it can be concluded that in oils there are permissible concentrations of simple acids formed during its operation.

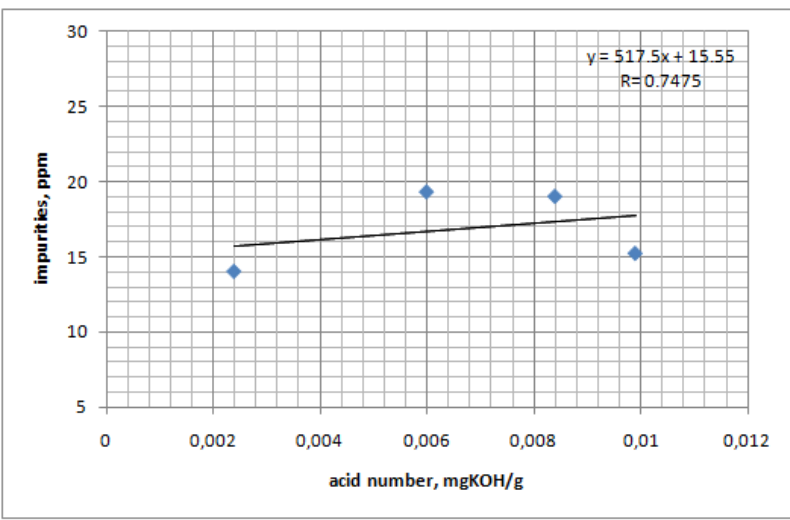

Fig. 1. The dependence of the impurity concentration on the acid number.

Breakdown voltage is a basic electrical characteristic that is very sensitive to the presence of impurities. The slightest change in the moisture content of a liquid dielectric, the presence of impurities in it sharply reduces the electrical strength [12]. A decrease in breakdown voltage indicates contamination of transformer oil with water, air, fibers, and other substances. The limiting moisture content that does not affect the breakdown voltage of $110-220 \mathrm{kV}$ transformers with $\mathrm{GK}$ oil is 30 ppm. Consequently, if the breakdown voltage decreases, and water is contained in permissible concentrations, this suggests that naphthenic acids and bases, substances that have a particular effect on this characteristic and react with Fisher's reagent, may be contained in the oil. Figure 2 shows the linear dependence of the impurity concentration on the breakdown voltage.

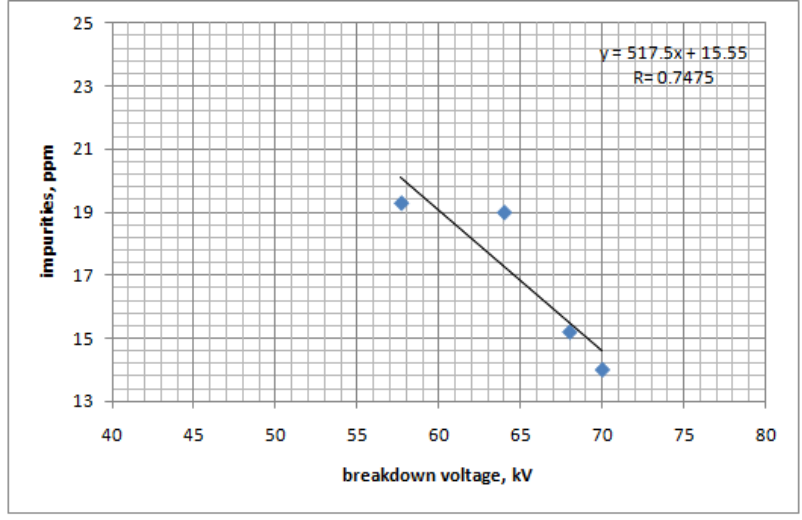

Fig. 2. The dependence of impurities on breakdown voltage.

The next characteristic of transformer oil that we study is the dielectric loss tangent tg I. In hermetic transformers containing aromatic hydrocarbons, an aging process of oil with hydrogen may be observed [13]. In parallel, coloring products appear that increase the tangent of dielectric losses.

The most stable are aromatic hydrocarbons with short side chains. The process of their oxidation takes place with the formation of compaction products. If the chain length increases, then the stability decreases. Hydrocarbons of the naphthenic-aromatic group are usually oxidized quite actively, forming a number of products, including resins. At the same time, an increased amount of resin leads to the formation of acidic products in oil, asphaltenes and carbenes [14, 15$]$.

If the conductivity of the samples under study is not determined by the presence of moisture, but by the amount of impurities, it can be concluded that the oil samples contain, within acceptable limits, acids, peroxides, aldehydes, alcohols and phenols, which affect the $\operatorname{tg} \delta$ value and interact with the Fisher reagent. Figures 3, 4 and 5 show the linear dependence of the impurity concentration on the tangent of dielectric loss angle at different temperatures. 


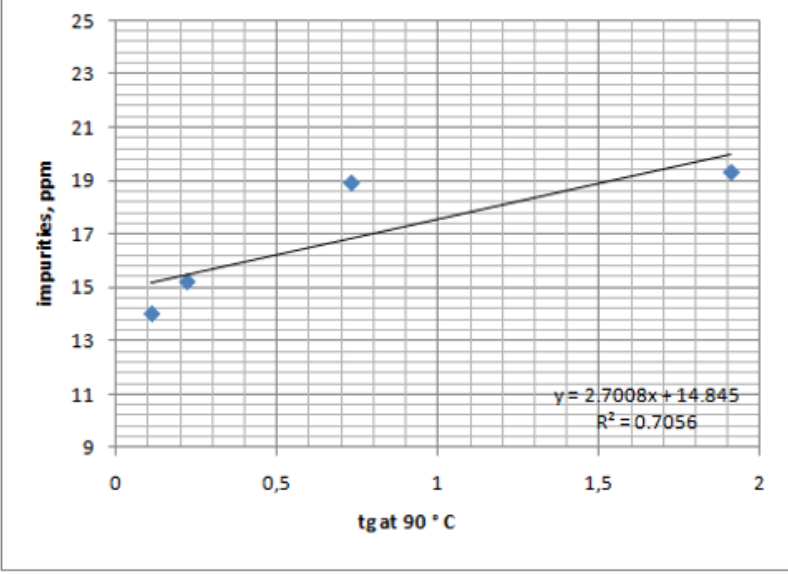

Fig. 3. Graph of impurities dependency in transformer oil from $\operatorname{tg} \delta$ at $90^{\circ} \mathrm{C}$.

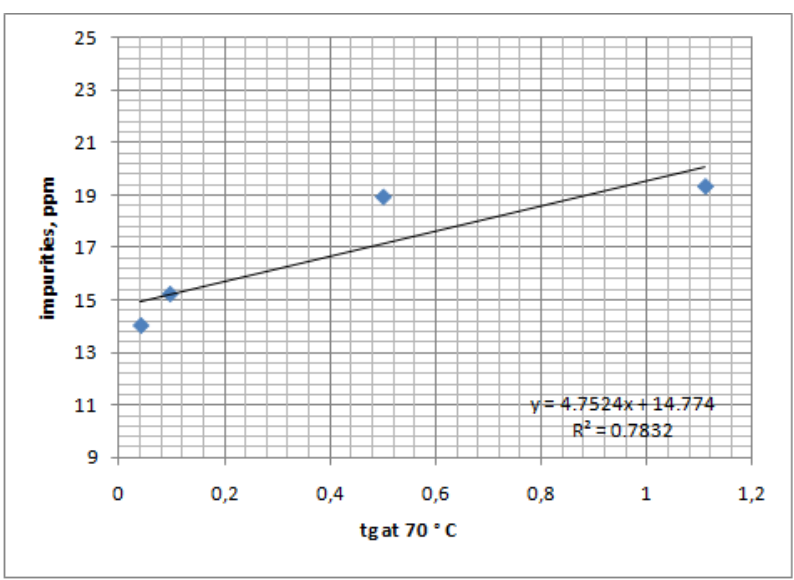

Fig. 4. Graph of impurities dependency in transformer oil from $\operatorname{tg} \delta$ at $70{ }^{\circ} \mathrm{C}$.

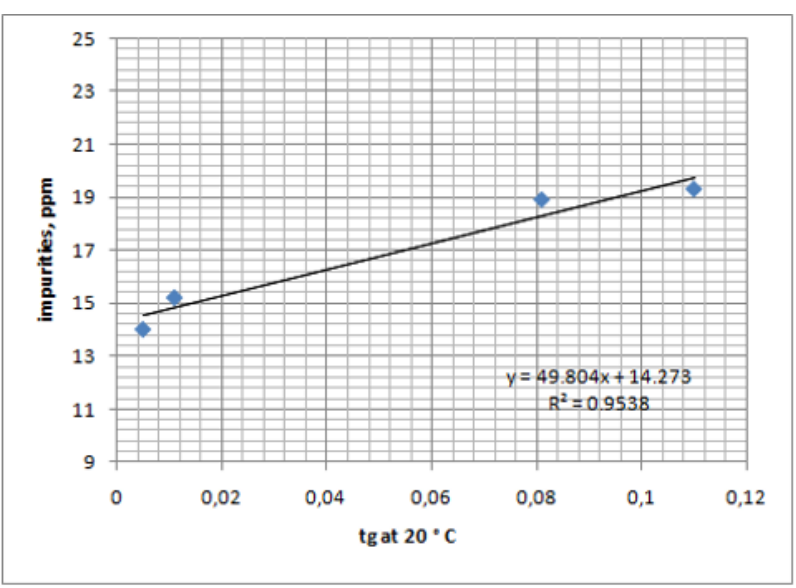

Fig. 5. Graph of impurities dependency in transformer oil from $\operatorname{tg} \delta$ at $20{ }^{\circ} \mathrm{C}$.

Thus, if all the characteristics are in permissible values, this indicates that there cannot be substances such as sulfur-containing compounds, resinous neutral substances, copper and iron salts, lacquer components of windings and old oil slurry in oil.

\section{Conclusions}

Currently, most modern methods for determining moisture content have certain errors in measurements. These errors are often dependent on impurities that are contained in the oil. Methods are proposed for removing moisture from transformer oil in order to further determine the absolute moisture content and amount of impurities. Experimental studies have shown that it is possible to determine both the amount of impurities and their nature in the used transformer oil.

\section{References}

1. B. Alekseev, Large Power Transformers, 88 (2010)

2. O. Kurakina, R. Arkhipov, B. Gizatullin, A. Shkalikov, O. Turanova, V. Kozlov, A. Turanov, Investigation of transformer oil by nuclear magnetic relaxation and Z-scanning, Chemistry and technology of fuels and oils, 1(49), 59-64 (2013)

3. S. Vysogorets, Development of methods for rapid analysis of the quality parameter of insulating oil, Reliability and safety of energy, 11(1), 41-47 (2018)

4. A. Syrtsov, D. Polovinka, O. Parsentev, Increasing the reliability of power transformers by improving their tests, Prospects for the development of information technology, 32, 76-86 (2016)

5. A. Kulikova, O. Turanova, V. Kozlov, A. Turanov, On the determination of the moisture content of transformer oil by Karl Fischer titration News of higher educational institutions, Energy problems, 11-12, 91-96 (2010)

6. S. Margolis, Effect of hydrocarbon composition on the measurement of water in oils by coulometric and volumetric Karl Fischer methods, Analytical Chemistry, 70(20), 4264 - 4270 (1998)

7. S. Margolis, Sources of systematic bias in the measurement of water by the coulometric and volumetric Karl Fischer methods, Analytical Chemistry, 69(23), 4864-4871 (1997)

8. R. Gilbert, J. Jalbert, P. Tetreault, Bias assessment of current technologies used for the determination of low levels of moisture in mineral oil samples, Analytical Chemistry, 73(3), 520-526 (2001)

9. V. Kozlov, I. Zagustina, Modified method for determining moisture-containing transformer oil News of higher educational institutions, Energy problems, 7-8, 87-90 (2016)

10. D. Valiullina, I. Zagustina, V. Kozlov, Determination of the qualitative composition of impurities in the used transformer oil, Bulletin of KGEU, 10-4 (40), 25-32 (2018)

11. N. Chernozhukov, Eds. S. Kerin, The Oxidation of Mineral Oils, 372 (1955)

12. R. Lipstein, Eds. M. Shakhnovich, Transformer Oil, 296 (1983)

13. O. Kurakina, V. Kozlov, O. Turanova, A. Turanov, Research of the changes in the structural group composition of transformer oil during operation, Problemele energeticii regionale, 2, 37 (2018) 
14. F. Unger, L. Andreeva, Eds. N. Krasnogorskaya, Fundamental Aspects of Petroleum Chemistry. The Nature of Tar and Asphaltenes, 192 (1995)

15. O. Turanova, A. Vendyukov, L. Gafiyatullin, V. Kozlov, Research of the mechanism of sediment formation in transformer oil, Chemistry and technology of fuels and oils, 4, 628-631 (2013) 